\title{
An Efficient and Secure Multidimensional Data Aggregation for Fog-Computing-Based Smart Grid
}

\author{
Omar Rafik Merad-Boudia ${ }^{(1)}$ and Sidi Mohammed Senouci ${ }^{(}$, Member, IEEE
}

\begin{abstract}
The secure multidimensional data aggregation (MDA) has been widely investigated in smart grid for smart cities. However, previous proposals use heavy computation operations either to encrypt or to decrypt the multidimensional data. Moreover, previous fault-tolerant mechanisms lead to an important computation cost, and also a high communication cost when considering a separate identification phase. In this article, we propose an efficient and secure MDA scheme, named ESMA. Unlike existing schemes, the multidimensional data in ESMA are structured and encrypted into a single Paillier ciphertext and thereafter, the data are efficiently decrypted. For privacy preserving, the Paillier cryptosystem is adopted in a fog computing-based architecture, and to achieve efficient authentication, the batch verification technique is applied. Besides, ESMA is fault tolerant, i.e., even if some of the smart meters fail to send their data, the final aggregation result will not be affected. Furthermore, ESMA can be adapted to respond to other queries than the summation of data. The performance analysis demonstrates the cost efficiency of ESMA both in computation and communication and the scalability as well. For instance, with a 16-bits size for each data type and 500 reporting smart meters, 40 data types can be supported in a single Paillier ciphertext. ESMA also resists various security attacks and preserves the user's privacy.
\end{abstract}

Index Terms-Data aggregation, fault tolerance (FT), fog computing, security and privacy, smart cities, smart grid (SG).

\section{INTRODUCTION}

$\mathbf{T}$ HE SMART grid (SG) network is the next-generation power grid network, which utilizes modern information and communication technologies and enables two-way communication between customers and service providers. Many electric utility companies have already substituted or introduced SG alongside the traditional power grid [1]. This gives electric utility companies the capability to perform fault diagnosis and to considerably enhance their power generation, distribution, transmission, and control. This can also avoid power blackouts and give the ability to anticipate power demands and facilitate the integration of renewable energy technologies in the grid [2], [3]. In SG, utility companies use

Omar Rafik Merad-Boudia is with the Computer Science Department, University of Oran 1 Ahmed Ben Bella, Oran 31000, Algeria, and also with the STIC Laboratory, University of Tlemcen, Tlemcen 13000, Algeria (e-mail: rafik.merad@univ-oran1.dz).

Sidi Mohammed Senouci is with the DRIVE Laboratory, University of Burgundy, 58000 Nevers, France (e-mail: sidi-mohammed.senouci@ u-bourgogne.fr). smart meters (SMs) to gather real-time consumption data and other information. The SM is considered as the most critical element placed at the user side. It enables customers to report their real-time consumption data (e.g., every quarter hour) to the control center (CC), and based on these data, the CC can anticipate power demands and adjust power generation, dynamic pricing, and so on. For instance, Kumari et al. [4] proposed a solution that accurately predicts future load consumption based on historical data using deep learning to save energy at the demand side and reduce energy production at the supply side of the system. However, SG is subject to several threats against security and privacy, e.g., by analyzing the real-time electricity usage data, an attacker would be able to threaten the user's privacy, and thus, inferring the user's life habits such as when a user exits the house. Consequently, some cryptographic techniques should be used to preserve the user's privacy [5].

The concept of data aggregation in the context of SG is introduced in [6]. A traditional data aggregation scheme considers a gateway node that aggregates the SMs reports and sends the result to the $\mathrm{CC}$, enabling the utility company to get the total consumption data. This gives the company the capability to dynamically adjust power distribution according to these data and to protect users' privacy as well. Recently, a fog computing architecture has been considered in SG for efficient aggregation [7]. With the help of such architecture, Nazmudeen et al. [8] proposed a framework for a distributed data aggregation. As stated in [9], traditional in-network aggregation' architectures face resource and scalability problems. They employ less powerful intermediate nodes and a large number of end nodes are usually connected with one intermediate node. By considering fog nodes (FNs), we can exploit FN's intrinsic abilities in terms of communication, computation, and storage, leading, therefore, to the optimal use of these nodes for aggregation purposes. This can also resolve the bandwidth and latency issues that arise in a cloud computing architecture [7].

Although previous solutions [6], [9]-[19] can prevent the disclosure of sensitive information to the adversary or the utility company, there are, however, some unresolved issues. First, the data aggregated are likely to be multidimensional (electricity consumption record, time, consumption purpose, etc.). Hence, more data provided more efficient is the analysis. Some works only enable one-dimensional aggregation (ODA), i.e., every dimension is processed separately. Moreover, existing multidimensional aggregation (MDA) schemes use 
complex cryptographic operations to encrypt or to decrypt the multidimensional data. Second, in some schemes, there is no mechanism for FT, in fact, in SG, SMs could malfunction for a certain amount of time and thus could not send their reports to the FN. In previous schemes, the CC has to ask FNs for the nonresponding SMs that leads to an important delay, and the aggregation process is then halted. Finally, previous schemes are only conceived to respond to the sum query. However, the CC may need other statistical functions than summation such as variance to further analyze power consumption. This could allow the detection of abnormal situations and load imbalance. Moreover, the CC may need to learn about the number of customers whose SM's reading is higher than a threshold, and also the total usage of these customers for predicting better power generation.

For these reasons, we propose ESMA, an Efficient and Secure Multidimensional data Aggregation for fog computingbased smart grid (FCSG). The main contributions of this article can be summarized as follows.

1) We propose an efficient MDA for FCSG. In ESMA, even if the data of each SM are multidimensional, the data reported to $\mathrm{FN}$ would still be a single Paillier ciphertext. Besides, we employ an encoding function that gives the $\mathrm{CC}$ the capability to efficiently recover the aggregated data from the ciphertext. The computation and communication resources are then conserved in comparison to previous schemes.

2) We employ the Paillier Cryptosystem and its homomorphic property to preserve user's privacy. So, the FN performs aggregation on ciphertexts, which prevents the disclosure of sensitive data. Also, we propose a faulttolerant mechanism to ensure that the final aggregated data recovered at $\mathrm{CC}$ are correct even if some nodes are malfunctioning.

3) We illustrate how ESMA can be adapted to answer other queries than summation. We also provide security analysis and performance evaluation to show the security and the efficiency of ESMA in comparison with the previous work.

The remainder of this article is organized as follows. Section II introduces the related work. Section III presents the system models and design goals. Section IV describes the preliminaries of ESMA before presenting its technical details in Section V. Section VI presents how ESMA can respond to other queries and Section VII discusses security analysis. Finally, Sections VIII and IX give the performance evaluation and conclusion, respectively.

\section{RELATED WORK}

In this section, we present an overview of traditional solutions to secure data aggregation in SG [10]-[14], and also the recently proposed ones in the context of FCSG [9], [16]-[19]. Introduced in [6], protecting the user's privacy via data aggregation has recently become one of the most attractive research topics. Li et al. [6] introduced secure in-network aggregation in the context of SG; they employ homomorphic encryption (HE) to avoid the disclosure of user's private data to intermediate aggregator nodes. Lu et al. [10] used Paillier HE and employed a super-increasing sequence to secure MDA, an additional exponentiation operation is computed for every dimension by SM. Boudia et al. [11] proposed an elliptic curve-based secure MDA using El Gamal HE with multiple public keys. Their scheme does not require complex cryptographic operations en route and leads to lower overhead. However, an additional elliptic curve scalar multiplication is computed for every dimension by SM. Ming et al. [12] and Zuo et al. [13] employed a super-increasing sequence with El Gamal HE to achieve MDA. In these two schemes, the $\mathrm{CC}$ has to solve the discrete logarithm problem (DLP) to retrieve the aggregated data. Badra and Zeadally [14] proposed a lightweight secure ODA using symmetric HE and DiffieHellman methods. Shen et al. [15] introduced the malicious data mining attack and proposed a secure ODA using Paillier $\mathrm{HE}$ and bilinear maps.

Recently, many solutions have been proposed in the context of FCSG. Lyu et al. [9] proposed a privacy-preserving fog-enabled aggregation scheme using one-time pad HE. The authors consider ODA and an FN that aggregates the collected data, decrypts, and then encrypts noisy aggregation. They propose a fault-tolerant mechanism to achieve resilience to node failures. However, an additional phase is required for this purpose, which leads to additional communication cost. Moreover, data integrity is not ensured in their scheme. Liu et al. [16] presented a fog-enabled aggregation scheme that employs a double trapdoor HE to encrypt one-dimensional data. Note that their scheme can achieve MDA by using a super-increasing sequence. However, similarly to the work in [10], an additional heavy computation cost will be added for every dimension. Okay et al. [17] proposed two efficient and lightweight secure aggregation protocols for FCSG. The authors employ Domingo-Ferrer HE and Paillier HE to encrypt ODA. They present mathematical models for these protocols and give extensive simulations and comparisons. Saleem et al. [18] proposed an efficient and privacy-preserving ODA for FCSG. The authors exploit a modified version of Paillier HE in order to encrypt the metering data and use MAC for DI. They propose a fault-tolerant mechanism to achieve resilience to node failures. However, the $\mathrm{CC}$ has to compute the discrete logarithm to retrieve the aggregated data. Furthermore, the fault-tolerant decryption cost increases with the increasing number of faulty SMs. Zhao et al. [19] proposed an ODA scheme for FCSG that achieves multifunctional statistics; the authors employ somewhat homomorphic encryption (SHE) to preserve the user's privacy. However, their proposal incurs high overheads in terms of communication and computation.

We compare in Table I the aforementioned works in terms of the data type (DT), the considered architecture (CA), the used technique (UT), fault tolerance (FT), source authentication (SA), and data integrity (DI). The authors of these works either consider data aggregation with one dimension [6], [9], [16]-[19] or use heavy computation operations to encrypt or to decrypt the multidimensional data [10]-[13]. Note that these schemes are only conceived to respond to the sum query. Also, the FT mechanisms proposed in some schemes, e.g., the scheme in [9], requires a separate 
TABLE I

Comparative AnALYsis of Previous Schemes

\begin{tabular}{cccccccc}
\hline \hline Scheme & DT & CA & UT & FT & PP & SA & DI \\
\hline Li et al. [6] & ODA & Cloud & Paillier & No & Yes & No & No \\
Lu et al. [10] & MDA & Cloud & Paillier & No & Yes & Yes & Yes \\
Merad et al. [11] & MDA & Cloud & ECEG & No & Yes & Yes & Yes \\
Ming et al. [12] & MDA & Cloud & ECEG & No & Yes & Yes & Yes \\
Zuo et al. [13] & MDA & Cloud & ElGamal & No & Yes & Yes & Yes \\
Badra et al. [14] & ODA & Cloud & Sym. HE & No & Yes & Yes & Yes \\
Sheng et al. [15] & ODA & Cloud & Paillier & No & Yes & Yes & Yes \\
Lyu et al. [9] & ODA & Fog & OTP & Yes & Yes & No & No \\
Liu et al. [16] & ODA & Fog & Trap. HE & No & Yes & No & No \\
Saleem et al. [18] & ODA & Fog & Paillier & Yes & Yes & Yes & Yes \\
Zhao et al. [19] & ODA & Fog & SHE & No & Yes & No & No \\
\hline \hline
\end{tabular}

phase to identify the faulty SMs and thus incurs a high communication cost. To overcome these drawbacks, we introduce in ESMA an encoding function to efficiently structure and secure the multidimensional data. ESMA can be adapted to respond to other queries than the summation of data without additional overhead. Moreover, the proposed FT mechanism preserves network resources.

\section{Models AND Design GoAL}

In this section, we define our network model, attacker model, and identify the design goal of ESMA.

\section{A. Network Model}

Similar to the previous work based on a fog computing architecture proposed for SG [7]-[9], [16]-[19], we consider in ESMA a three-level system. Fig. 1 shows a large number of SMs in the first level, FNs in the second level, and the $\mathrm{CC}$ in the third level. We consider that the CC covers $k_{2}$ FNs and each FN in turn covers $k_{1}$ SMs. The CC is responsible for collecting the users' data to anticipate power demands and to adjust power generation, dynamic pricing, and so on. Each $\mathrm{SM}_{i j}\left(i=1, \ldots, k_{1}, j=1, \ldots, k_{2}\right)$ collects the multidimensional data, performs cryptographic operations, and sends a report to the corresponding $\mathrm{FN}_{j}$. Once received, the reports are homomorphically aggregated by $\mathrm{FN}_{j}$. The result is then forwarded to the CC. After the reception of the $\mathrm{FN}_{j}$ ' report, the $\mathrm{CC}$ performs decryption and gets the aggregation result. This can help the CC to make appropriate decisions. For generating the public/private keys used in the Paillier cryptosystem and other secret parameters, we consider a trust authority (TA), which is a fully reliable entity. After providing the corresponding secret parameters to all of the entities in the system, TA will no longer be required in the MDA process.

\section{B. Attacker Model}

In our attacker model, we consider that FNs and $\mathrm{CC}$ are honest-and-curious, i.e., they follow the protocol faithfully but are curious about the multidimensional data contained in the reports, e.g., by keeping all of the reported data to optimize the chance to retrieve user's private data. Besides, we consider that the users are honest and will not report false consumption data. However, there exists an external adversary $\mathcal{A}$ that can eavesdrop the communication flows and try to identify the report's content. $\mathcal{A}$ can also launch active attacks, such as

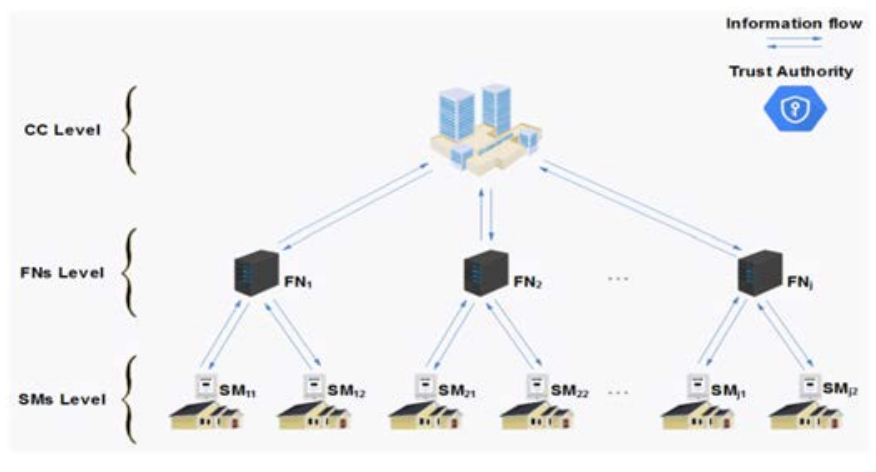

Fig. 1. System model.

data modification, false data injection, replay, or stealing the user's private data by intruding the database of CC and FN.

In addition to the aforementioned attacks and due to the SM wastage, we also assume that certain SMs could be in failure status and thereby will fail to send their data to FN.

\section{Design Goal}

Our design goal is to propose a fault-tolerant, efficient, and privacy-preserving MDA scheme for FCSG such that the CC can obtain multiple real-time data from one single aggregated data. Specifically, the following goals should be satisfied.

1) The user's private data cannot be compromised by $\mathcal{A}$. The $\mathrm{CC}$ is the only entity that can read the aggregated multidimensional data in ESMA, and no one can have access to the sensitive individual data, including the CC.

2) FNs and CC should be able to verify the authenticity of the received reports. They should also detect if a report has been modified during the transmission and comes really from the legal entity.

3) The proposed scheme should be fault tolerant. When certain SMs fail to communicate their data, they should be detected by the corresponding FN and reported to the CC.

4) The computation cost of cryptographic operations performed at SM, FN, and CC should be efficient. Moreover, the multidimensional data should be encrypted into one single ciphertext to reduce the cost of communication.

\section{Preliminaries}

In this section, we briefly introduce the security techniques used in ESMA, such as the Paillier homomorphic cryptosystem and bilinear maps.

\section{A. Paillier Homomorphic Cryptosystem}

The Paillier homomorphic cryptosystem is a classic encryption [20] that can achieve additive homomorphism property. The encryption is proved to be semantically secure against chosen-plaintext attacks. Thus, it has been widely exploited in secure data aggregation protocols (see Table I). The key generation, encryption, and decryption processes operate as follows.

1) Key Generation: Given a security parameter $\kappa$, two $\kappa$ bit prime numbers $p_{0}$ and $q_{0}$ are randomly selected. Let the 
TABLE II

NOTATIONS

\begin{tabular}{ll}
\hline \hline Notation & Definition \\
\hline ODA & One-dimensional aggregation \\
MDA & Multidimensional aggregation \\
$\mathrm{CC}$ & The control center \\
$\mathrm{FN}$ & The fog node \\
$\mathrm{SM}$ & The smart meter \\
$\mathrm{DLP}$ & Discrete Logarithm Problem \\
ECDLP & Elliptic Curve Discrete Logarithm Problem \\
$\mathrm{n}$ & The modulus $\mathrm{n}=\mathrm{p}_{\mathrm{o}} \mathrm{q}_{0}$ \\
$\mathrm{~g}$ & The generator of $\mathbb{Z}_{n^{2}}$ \\
$\mathbb{G}_{1}$ & An additive group \\
$\mathbb{G}_{2}$ & A multiplicative group \\
$(n, g)$ & The public key pair \\
$(\lambda, \mu)$ & The private key pair \\
$e$ & A bilinear pairing \\
$\mathrm{H}$ & A secure hash function, $H:\{0,1\}^{*} \rightarrow \mathbb{G}_{1}$ \\
$\mathrm{k}_{1}$ & The number of $\mathrm{SM} \mathrm{covered} \mathrm{by} \mathrm{one} \mathrm{FN}$ \\
$\mathrm{k}_{2}$ & The number of $\mathrm{FN}$ covered by CC \\
$x_{i j}$ & The secret share of $\mathrm{SM}_{\mathrm{ij}}$ \\
$s k_{i j}$ & The secret key of $\mathrm{SM}_{\mathrm{ij}}$ \\
$p k_{i j}$ & The public key of $\mathrm{SM}_{\mathrm{ij}}$ \\
$\mathrm{m}_{\mathrm{i} l}$ & The data type $l$ of $\mathrm{SM}_{\mathrm{ij}}$ \\
$\mathrm{d}_{\mathrm{i} l}$ & The encoded form of $\mathrm{m}_{\mathrm{i} l}$ \\
$l$ & The number of data types \\
$2^{\mathrm{z}}$ & The maximum value of a data type \\
\hline \hline
\end{tabular}

RSA modulus $n=p_{0} q_{0}$ and $\lambda=\operatorname{lcm}\left(p_{0}-1, q_{0}-1\right)$.We define a function $L(u)=u-1 / n$ and calculate $\mu=$ $\left(L\left(g^{\lambda} \bmod n^{2}\right)\right)^{-1} \bmod n$, where $g$ is the generator of $\mathbb{Z}_{n^{2}}^{*}$ such that $\operatorname{gcd}\left(L\left(g^{\lambda} \bmod n^{2}\right), n\right)=1$. The public key is the tuple $(n, g)$ and the corresponding private key is the tuple $(\lambda, \mu)$.

2) Encryption: Choose a random number $r \in \mathbb{Z}_{n}^{*}$ to encrypt a given message $m \in \mathbb{Z}_{n}: c=E(m)=g^{m} \cdot r^{n} \bmod n^{2}$.

3) Decryption: For the ciphertext $c$, the corresponding plaintext is calculated by $m=D(c)=$ $L\left(c^{\lambda} \bmod n^{2}\right) \mu \bmod n$.

\section{B. Bilinear Maps}

Let $\mathbb{G}_{1}$ be an additive group and $\mathbb{G}_{2}$ be a multiplicative group, both of prime order $q$, i.e., $\left|\mathbb{G}_{1}\right|=\left|\mathbb{G}_{2}\right|=q$. A bilinear map $e: \mathbb{G}_{1} \times \mathbb{G}_{1}=\mathbb{G}_{2}$, which has the following properties.

1) Bilinearity: $\forall P, Q \in \mathbb{G}_{1}$ and $\forall a, b \in \mathbb{Z}_{q}^{*}$, $e(a P, b Q)=e(P, Q)^{a b}$

2) Non Degeneracy: $\exists P \in \mathbb{G}_{1}$ where $e(P, Q) \neq 1_{\mathbb{G} 2}$

3) Computability: $\forall P, Q \in \mathbb{G}_{1}$, there exists an efficient algorithm to compute $e(P, Q)$ in polynomial time.

We use the notation Gen to denote the algorithm, taken as input a security parameter $\kappa_{1}$, which outputs a 5 -tuple $(q$, $P, \mathbb{G}_{1}, \mathbb{G}_{2}, e$ ), where $q$ is $\kappa_{1}$-bit prime, $\mathbb{G}_{1}$ and $\mathbb{G}_{2}$ are two cyclic groups with order $q, P \in \mathbb{G}_{1}$ is a generator, and $e$ is a nondegenerated and computable bilinear map.

\section{OUR Proposed ESMA SCHEME}

In this section, we present ESMA for FCSG; it mainly consists of five main parts: 1) system initialization; 2) registration; 3) SM report generation; 4) data aggregation; and 5) data reading. A list of acronyms and symbols used in this article along with their meaning is shown in Table II.

\section{A. System Initialization}

For the fog-based SG system under consideration, we suppose that TA bootstraps the whole system and in charge of system parameters configuration. The parameter generation and the secret shares generation processes operate as follows.

1) Parameter Generation: Besides producing two security parameters $\left(\kappa, \kappa_{1}\right)$, as mentioned in Section IV, the TA randomly selects two $\kappa$-bit large prime numbers $p_{0}$ and $q_{0}$ and computes $n=p_{0} q_{0}$ and $\lambda=\operatorname{lcm}\left(p_{0}-1, q_{0}-\right.$ 1). Then, the TA constructs a function $L(u)=u-1 / n$ and calculates $\mu=\left(L\left(g^{\lambda} \bmod n^{2}\right)\right)^{-1} \bmod n$, where $g$ is the generator of $\mathbb{Z}_{n^{2}}^{*}$ such that $\operatorname{gcd}\left(L\left(g^{\lambda} \bmod n^{2}\right)\right.$, $n)=1$. The public key is the tuple $(n, g)$ and the corresponding private key is the tuple $(\lambda, \mu)$. Furthermore, given $\kappa_{1}$, TA generates the bilinear parameters $\left(q, P, \mathbb{G}_{1}\right.$, $\left.\mathbb{G}_{2}, e\right)$ by running $\operatorname{Gen}\left(\kappa_{1}\right)$. Then, TA defines a secure cryptographic hash function $H:\{0,1\}^{*} \rightarrow \mathbb{G}_{1}$.

2) Secret Shares Generation: Using a pseudorandom number generator function, TA generates $k_{1} * k_{2}$ secret shares $x_{i j} \in \mathbb{Z}_{n}^{*},\left(i=1, \ldots, k_{1}, j=1, \ldots, k_{2}\right)$ and computes $x_{0 j}$ such that

$$
x_{0 j}=\sum_{i=1}^{k_{1}} x_{i j} \bmod n, \text { where } j=1, \ldots, k_{2} .
$$

Note that the adversary cannot calculate $x_{0 j}$ without knowing all secret shares $x_{i j}$.

\section{B. Registration}

All $\mathrm{SM}_{i j}, \mathrm{FN}_{j}$, and $\mathrm{CC}$ need to be registered in the TA, we illustrate this phase as follows.

1) Registration of $S M_{i j}$ : The $\mathrm{SM}_{i j}$ (for all $i \in\left\{1,2, \ldots, k_{1}\right\}$ ) first chooses an identity $\mathrm{ID}_{i j}$. Then, TA randomly chooses a number $s k_{i j} \in \mathbb{Z}_{q}^{*}$ and computes $p k_{i j}=s k_{i j} P$, and securely delivers $s k_{i j}$ to $\mathrm{SM}_{i j}$. Moreover, TA transmits the secret $x_{i j}$.

2) Registration of $F N_{j}$ : The $\mathrm{FN}_{j}$ (for all $j \in\left\{1,2, \ldots, k_{2}\right\}$ ) first chooses an identity $\mathrm{ID}_{j}$. Then, TA randomly chooses a number $s k_{j} \in \mathbb{Z}_{q}^{*}$ and computes $p k_{j}=s k_{j} P$. Finally, the TA securely delivers $s k_{j}$ to $\mathrm{FN}_{j}$.

3) Registration of $C C$ : The $\mathrm{CC}$ first chooses an identity $\mathrm{ID}_{\mathrm{CC}}$. Then, TA transmits the tuple $(\lambda, \mu)$, and the secret shares $x_{i j}$ along with $x_{0 j}$.

At the end of this phase, TA publishes the system parameters as $\left\{e, q, n, g, P, p k_{i j}, p k_{j}, \mathbb{G}_{1}, \mathbb{G}_{2}, H\right\}$.

\section{Smart Meter Report Generation}

As the client's consumption information is periodically reported to FN, e.g., every quarter hour, to protect consumer privacy from exposure, an SM needs to encrypt such private information. Thus, each $\mathrm{SM}_{i j}$ measures and generates its $l$ types of data $\left(m_{i 1}, m_{i 2}, \ldots, m_{i l}\right)$. The following specific steps are performed.

Step-1: Let $z$ be the maximum number of bits that could represent a DT $m, \mathrm{SM}_{i j}$ encodes its $l$ types of data $\left(m_{i 1}, m_{i 2}, \ldots, m_{i l}\right)$ into $\left(d_{i 1}, d_{i 2}, \ldots, d_{i l}\right)$ and constructs 
$\overline{D_{i j}}$ as follows:

$$
\begin{aligned}
d_{i k} & =\left(m_{i k}\right)_{2} \| 0^{\theta}, k=1, \ldots, l \\
\text { where } \theta & =\left(\left\lceil\log _{2}\left(k_{1}\right)\right\rceil+z\right) *(k-1) \\
D_{i j} & =d_{i 1}+d_{i 2}+\cdots+d_{i l} .
\end{aligned}
$$

After encoding, $\mathrm{SM}_{i j}$ computes $\overline{D_{i j}}$

$$
\overline{D_{i j}}=D_{i j}+x_{i j} \bmod n .
$$

Step-2: $\mathrm{SM}_{i j}$ chooses a random number $r_{i j} \in \mathbb{Z}_{n}^{*}$, and computes the ciphertext as follows:

$$
C_{i j}=g^{\overline{D_{i j}}} \cdot r_{i j}^{n} \bmod n^{2} .
$$

Step-3: $\mathrm{SM}_{i j}$ uses its private key $s k_{i j}$ to compute the signature as follows:

$$
\sigma_{i j}=s k_{i j} H\left(C_{i j}\left\|\mathrm{ID}_{i j}\right\| \mathrm{TS}\right)
$$

where TS is the current timestamp.

Step-4: $\mathbf{S M}_{i j}$ sends, to the corresponding $\mathrm{FN}_{j}$, the data packet that contains $\left\{C_{i j}\right.$, $\mathrm{ID}_{i j}$, TS, $\left.\sigma_{i j}\right\}$. It is necessary to stress that every $\mathrm{SM}_{i j}$ should produce and encrypt a message $D_{i j}$ as above. An example with $l=3$ is depicted in Fig. 2.

\section{Data Aggregation}

This part consists of two possible phases. In the case if all of the $\mathrm{SM}_{i j}$ send their reports, $\mathrm{FN}_{j}$ will perform an all-inclusive data aggregation; otherwise, it will perform a fault-tolerant data aggregation.

1) All-Inclusive Data Aggregation: If all of the $\mathrm{SM}_{i j}$ report their data to the corresponding $\mathrm{FN}_{j}, \mathrm{FN}_{j}$ will first perform the batch verification to verify the received signatures, i.e., check whether

$$
e\left(P, \sum_{i=1}^{k_{1}} \sigma_{i j}\right)=\prod_{i=1}^{k_{1}} e\left(p k_{i j}, H\left(C_{i j}\left\|\mathrm{ID}_{i j}\right\| \mathrm{TS}\right)\right) .
$$

If the equation does hold, it means the signatures are valid. The correctness is as follows:

$$
\begin{aligned}
e\left(P, \sum_{i=1}^{k_{1}} \sigma_{i j}\right) & =e\left(P, \sum_{i=1}^{k_{1}} s k_{i j} H\left(C_{i j}\left\|\mathrm{ID}_{i j}\right\| \mathrm{TS}\right)\right) \\
& =\prod_{i=1}^{k_{1}} e\left(P, s k_{i j} H\left(C_{i j}\left\|\mathrm{ID}_{i j}\right\| \mathrm{TS}\right)\right) \\
& =\prod_{i=1}^{k_{1}} e\left(p k_{i j}, H\left(C_{i j}\left\|\mathrm{ID}_{i j}\right\| \mathrm{TS}\right)\right) .
\end{aligned}
$$

Note that the batch verification reduces the number of pairing operations from $2 k_{1}$ to $k_{1}+1$. After checking the validity, $\mathrm{FN}_{j}$ aggregates all the ciphertexts and sends the aggregated message to the CC. The following steps are performed.

Step-1: $\mathrm{FN}_{j}$ aggregates the $k_{1}$ encrypted ciphertexts as

$$
C_{j}=\prod_{i=1}^{k_{1}} C_{i j} \bmod n^{2}
$$

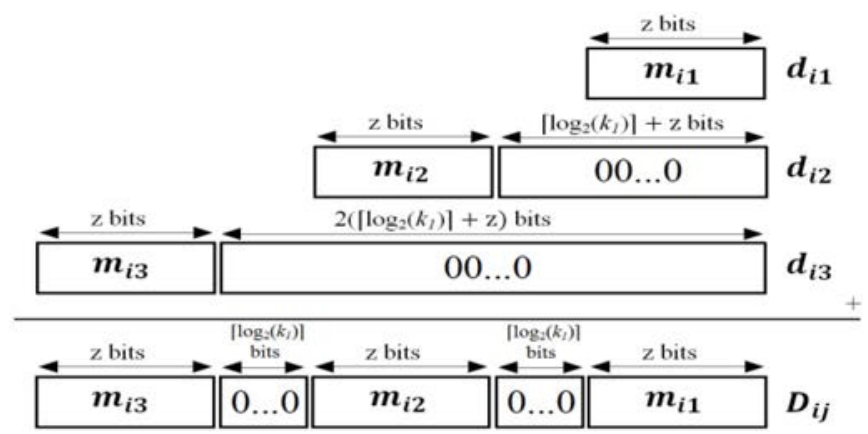

Fig. 2. Example of $D_{i j}$ with $l=3$.

$$
\begin{aligned}
& =\prod_{i=1}^{k_{1}} g^{\overline{D_{i j}}} \cdot r_{i j}^{n} \bmod n^{2} \\
& =g^{\sum_{i=1}^{k_{1}} \overline{D_{i j}}} \cdot\left(\prod_{i=1}^{k_{1}} r_{i j}\right)^{n} \bmod n^{2} \\
& =g^{\sum_{i=1}^{k_{1}}\left(D_{i j}+x_{i j}\right) \bmod n} \cdot\left(\prod_{i=1}^{k_{1}} r_{i j}\right)^{n} \bmod n^{2} .
\end{aligned}
$$

Step-2: $\mathrm{FN}_{j}$ uses its private key $s k_{j}$ to compute the signature as follows:

$$
\sigma_{j}=s k_{j} H\left(C_{j}\left\|\mathrm{ID}_{j}\right\| \mathrm{TS}\right)
$$

where TS is the current timestamp.

Step-3: $\mathrm{FN}_{j}$ sends, to the $\mathrm{CC}$, an all-inclusive report that contains $\left\{C_{j}, \mathrm{ID}_{j}, \mathrm{TS}, \sigma_{j}\right\}$.

2) Fault-Tolerant Data Aggregation: If some SMs breakdown, $\mathrm{FN}_{j}$ will not receive the corresponding packets. Let $U_{i j}$ be the set of all legitimate SM devices and $U_{i j}^{\prime}$ be the set of failed SM devices $\left(U_{i j}^{\prime} \in U_{i j}\right)$. Consequently, we have

$$
x_{0 j} \neq \sum_{i \in U_{i j} / U_{i j}^{\prime}} x_{i j} \bmod n .
$$

Thus, this phenomenon will directly affect the correctness of the final decryption result. $\mathrm{FN}_{j}$ needs to send the set $U_{i j}^{\prime}$ to the $\mathrm{CC}$. Therefore, in this case, $\mathrm{FN}_{j}$ first validates the received signatures, i.e., check whether

$$
e\left(P, \sum_{i \in U_{i j} / U_{i j}^{\prime}} \sigma_{i j}\right)=\prod_{i \in U_{i j} / U_{i j}^{\prime}} e\left(p k_{i j}, H\left(C_{i j}\left\|\mathrm{ID}_{i j}\right\| \mathrm{TS}\right)\right) .
$$

If the equation does hold, it means the signatures are valid. Then, it performs aggregation on the received ciphertexts and computes the fault-tolerant aggregated ciphertext. The following specific steps are performed.

Step-1: $\mathrm{FN}_{j}$ aggregates the received ciphertexts as

$$
\begin{aligned}
C_{j}^{\prime} & =\prod_{i \in U_{i j} / U_{i j}^{\prime}} C_{i j} \bmod n^{2} \\
& =g^{\sum_{i \in U_{i j} / U_{i j}^{\prime}}\left(D_{i j}+x_{i j}\right) \bmod n} \cdot\left(\prod_{i \in U_{i j} / U_{i j}^{\prime}} r_{i j}\right)^{n} \bmod n^{2} .
\end{aligned}
$$


Step-2: $\mathrm{FN}_{j}$ uses its private key $s k_{j}$ to compute the signature as follows:

$$
\sigma_{j}^{\prime}=s k_{j} H\left(C_{j}^{\prime}\left\|\mathrm{ID}_{j}\right\| \mathrm{TS}\right)
$$

where TS is the current timestamp.

Step-3: $\mathrm{FN}_{j}$ sends, to the $\mathrm{CC}$, a fault-tolerant report that contains $\left\{C_{j}^{\prime}, \mathrm{ID}_{j}, \mathrm{TS}, \sigma_{j}^{\prime}, U_{i j}^{\prime}\right\}$.

\section{E. Data Reading}

This part consists of two possible phases. If the CC receives an all-inclusive report from $\mathrm{FN}_{j}$, it will perform an all-inclusive data reading; otherwise, it will perform a faulttolerant data reading.

1) All-Inclusive Data Reading: Upon receiving the allinclusive report from $\mathrm{FN}_{j}$, CC first verifies the signature according to the following equation:

$$
e\left(P, \sigma_{j}\right)=e\left(p k_{j}, H\left(C_{j}\left\|\mathrm{ID}_{j}\right\| \mathrm{TS}\right)\right) .
$$

If the equation does hold, it means the signatures are valid. After checking the validity, $\mathrm{CC}$ decrypts the aggregated ciphertext $C_{j}$ and retrieves the aggregated data by performing the following steps.

Step-1: CC decrypts the aggregated ciphertext $C_{j}$. From (8), by taking

$$
M=\sum_{i=1}^{k_{1}}\left(D_{i j}+x_{i j}\right) \bmod n, \text { and } R=\prod_{i=1}^{k_{1}} r_{i j} .
$$

The report $g^{M} \cdot R^{n} \bmod n^{2}$ is still a ciphertext of the Paillier Cryptosystem. Thus, $\mathrm{CC}$ uses the tuple $(\lambda, \mu)$ to recover $M$ as

$$
M=L\left(C_{j}^{\lambda} \bmod n^{2}\right) \mu \bmod n .
$$

Step-2: After decrypting, $\mathrm{CC}$ uses $x_{0 j}$ to obtain $\sum_{i=1}^{k_{1}} D_{i j}$ as

$$
\sum_{i=1}^{k_{1}} D_{i j}=M-x_{0 j} \bmod n .
$$

Step-3: CC uses the decoding function to retrieve each aggregated data $\sum_{i=1}^{k_{1}} d_{i k}$. CC divides the binary representation of $\sum_{i=1}^{k_{1}} D_{i j}$ into $l$ blocks of bits, the length of each block is $\left(\left\lceil\log _{2}\left(k_{1}\right)\right\rceil+z\right)$. Thus, the first $\left(\left\lceil\log _{2}\left(k_{1}\right)\right\rceil+z\right)$ least significant bits correspond to the aggregation result $\sum_{i=1}^{k_{1}} m_{i 1}$ and so on. The CC then retrieves the aggregation result of each type of data as

$$
\left(\sum_{i=1}^{k_{1}} D_{i j}\right)_{2}=\sum_{i=1}^{k_{1}} m_{i l}\|\ldots . .\| \sum_{i=1}^{k_{1}} m_{i 2} \| \sum_{i=1}^{k_{1}} m_{i 1} .
$$

Due to overflow during aggregation, the required extra bits cannot be more than $\left\lceil\log _{2}\left(k_{1}\right)\right\rceil$ when $k_{1}$ numbers are added. This explains why we choose to $\operatorname{pad}\left(\left\lceil\log _{2}\left(k_{1}\right)\right\rceil+z\right)^{*}(k-$ 1) zeros after $m_{i k}$ in every $d_{i k}$. Fig. 3. shows the aggregated data retrieved by the CC when $k_{1}=4$ and $l=3$, where $D_{i j}$ was generated by $\mathrm{SM}_{i j}$.

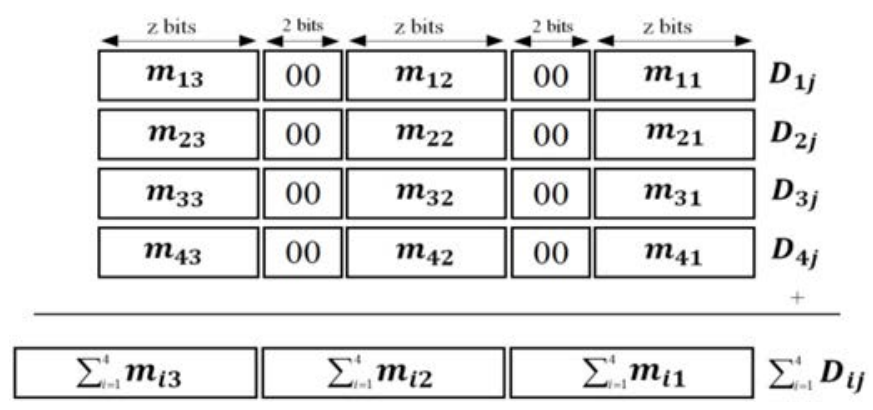

Fig. 3. Example with $k_{1}=4$ and $l=3$.

2) Fault-Tolerant Data Reading: Upon receiving the faulttolerant report from $\mathrm{FN}_{j}, \mathrm{CC}$ first verifies the signature according to the following equation:

$$
e\left(P, \sigma_{j}^{\prime}\right)=e\left(p k_{j}, H\left(C_{j}^{\prime}\left\|\mathrm{ID}_{j}\right\| \mathrm{TS}\right)\right) .
$$

If the equation does hold, it means the signatures are valid. $\mathrm{CC}$ decrypts the aggregated ciphertext $C_{j}^{\prime}$ and retrieves the aggregated data by performing the following steps.

Step-1: CC decrypts the aggregated ciphertext $C_{j}^{\prime}$ as

$$
M^{\prime}=L\left(C_{j}^{\prime \lambda} \bmod n^{2}\right) \mu \bmod n .
$$

Step-2: After decrypting, $\mathrm{CC}$ computes $x_{0 j}^{\prime}$ as

$$
x_{0 j}^{\prime}=\sum_{i \in U_{i j} / U_{i j}^{\prime}} x_{i j} \bmod n .
$$

Step-3: CC uses $x_{0 j}^{\prime}$ to obtain the fault-tolerant aggregated multidimensional data

$$
\sum_{i \in U_{i j} / U_{i j}^{\prime}} D_{i j}^{\prime}=M^{\prime}-x_{0 j}^{\prime} \bmod n
$$

Step-4: CC uses the decoding function, as described earlier, to retrieve the fault-tolerant aggregation result of each type of data as

$$
\begin{gathered}
\left(\sum_{i \in U_{i j} / U_{i j}^{\prime}} D_{i j}^{\prime}\right)_{2}=\sum_{i \in U_{i j} / U_{i j}^{\prime}} m_{i l}^{\prime}\|\cdots \cdot\| \sum_{i \in U_{i j} / U_{i j}^{\prime}} m_{i 1}^{\prime} . \\
m_{i 2}^{\prime} .
\end{gathered}
$$

\section{Answering Other Queries}

In this section, we discuss how to adapt ESMA to answer other queries, namely, variance report query and set-aggregation report query.

\section{A. Variance Report Query}

The CC may need other statistical functions than summation such as variance to further analyze power consumption. This could allow the detection of abnormal situations and load imbalance. In the following, we present how ESMA can be adapted to achieve dual-functional MDA for FCSG, which 
can support, for each type of data, aggregation of mean and variance at the same time. Observe that

$$
\begin{aligned}
\operatorname{VAR}\left(m_{i 1}\right) & =\frac{\sum_{i=1}^{k_{1}} m_{i 1}^{2}}{k_{1}}-\left(\frac{\sum_{i=1}^{k_{1}} m_{i 1}}{k_{1}}\right)^{2} \\
& =\frac{1}{k_{1}} \sum_{i=1}^{k_{1}} m_{i 1}^{2}-\frac{1}{k_{1}^{2}}\left(\sum_{i=1}^{k_{1}} m_{i 1}\right)^{2} .
\end{aligned}
$$

If the $\mathrm{CC}$ needs to perform a variance analysis on multidimensional data, each $\mathrm{SM}_{i j}$ add the data $\left(m_{i 1}^{2}\right.$, $\left.m_{i 2}^{2}, \ldots, m_{i l}^{2}\right)$ in addition to $\left(m_{i 1}, m_{i 2}, \ldots, m_{i l}\right)$ and encode them as follows:

$$
d_{i k}=\left(m_{i k}^{2}\right)_{2}\left\|\left(m_{i k}\right)_{2}\right\| 0^{\theta}, k=1, \ldots, l
$$

where $\theta=\left(2\left\lceil\log _{2}\left(k_{1}\right)\right\rceil+3 z\right) *(k-1)$ and $\left(m_{i k}\right)_{2}$ is padded by zeros in the left to be represented in $\left(\left\lceil\log _{2}\left(k_{1}\right)\right\rceil+z\right)$ bits.

Due to square calculation, the required bits for $m_{i k}^{2}$ cannot be more than $2 z$ where $z$ is the maximum number of bits that could represent a DT. This explains why we choose to pad $\left(2\left\lceil\log _{2}\left(k_{1}\right)\right\rceil+3 z\right) *(k-1)$ zeros in every $d_{i k}$. The $\mathrm{SM}_{i j}$ then follows the same steps as previously described to produce its report. At the end of the decryption phase, $\mathrm{CC}$ retrieves the queried data as follows:

$$
\left(\sum_{i=1}^{k_{1}} D_{i j}\right)_{2}=\sum_{i=1}^{k_{1}} m_{i l}^{2}\left\|\sum_{i=1}^{k_{1}} m_{i l}\right\| \cdots \cdots\left\|\sum_{i=1}^{k_{1}} m_{i 1}^{2}\right\| \sum_{i=1}^{k_{1}} m_{i 1} .
$$

\section{B. Set Aggregation Query}

We suppose that there exist $f$ subsets corresponding to $f$ electricity consumption ranges, such that $\left[R_{1}, R_{2}\right),\left[R_{2}\right.$, $\left.R_{3}\right), \ldots,\left[R_{f},+\infty\right)$. The CC may need to calculate the total consumption and also the number of users of each subset in a period [21]. In the following, we show how ESMA can be adapted to achieve the data aggregation of multisubset for FCSG. We assume that if the electricity consumption data, generated by $\mathrm{SM}_{i j}, m_{i} \in\left[R_{S}, R_{s+1}\right), \mathrm{SM}_{i j}$ will lie in the subset $\mathbb{U}_{s} \subset \mathbb{U}$, where $\mathbb{U}=\mathbb{U}_{1} \cup \mathbb{U}_{2} \cup \cdots \cup \mathbb{U}_{f}$, and $\mathbb{U}_{s} \cap \mathbb{U}_{t}=\emptyset$, for $s$, $t=1,2, \ldots, f, s \neq t$. We also have $E=E_{1}+E_{2}+\cdots+E_{f}, E_{1}$ is the total electricity consumption of the subset $\mathbb{U}_{1}$ and so on. We can then consider for each subset a block of bits in our encoding function. Each $\mathrm{SM}_{i j}$ encodes its data $m_{i} \in\left[R_{S}, R_{S+1}\right)$ into $D_{i j}$ as follows:

$$
D_{i j}=1\left\|\left(m_{i}\right)_{2}\right\| 0^{\theta}, s=1, \ldots, f
$$

where $\theta=\left(2 *\left\lceil\log _{2}\left(k_{1}\right)\right\rceil+z\right) *(s-1)$ and $\left(m_{i}\right)_{2}$ is padded by zeros in the left to be represented in $\left(\left\lceil\log _{2}\left(k_{1}\right)\right\rceil+z\right)$ bits.

The $\mathrm{SM}_{i j}$ then follows the same steps as previously described to produce its report. Afterward, $\mathrm{FN}_{j}$ aggregates the number of users and total usage of each subset. At the end of the decryption phase, $\mathrm{CC}$ retrieves the queried data as follows:

$$
\left(\sum_{i=1}^{k_{1}} D_{i j}\right)_{2}=\left|\mathbb{U}_{f}\right|\left\|E_{f}\right\| \cdots\left|\mathbb{U}_{2}\right|\left\|E_{2}\right\|\left|\mathbb{U}_{1}\right| \| E_{1}
$$

\section{SECURITY ANALYSIS}

In this section, we analyze the security of ESMA. In particular, we show how ESMA can protect the user's individual data, and ensure data integrity and source authentication.

\section{A. Privacy Protection}

In the SM report generation phase, the sensitive multidimensional data $d_{i j}$ is blinded by adding the secret key $x_{i j}$ modulo $n$ to get the ciphertext: $C_{i j}=g^{d_{i j}+x_{i j} \bmod n}$. $r^{n} \bmod n^{2} \cdot \mathrm{SM}_{i j}$ sends $C_{i j}$ to the corresponding $\mathrm{FN}_{j}$ instead of $d_{i j}$ directly. The $\mathrm{FN}_{j}$ is then unable to decrypt the received ciphertext and to read the sensitive data $d_{i j}$ without knowing the Paillier private key and $x_{i j}$. The Paillier cryptosystem is semantically secure, so the encryption is secure against any form of ciphertext analysis. User's multidimensional data $\left(m_{i 1}, m_{i 2}, \ldots, m_{i l}\right)$ in $d_{i j}$ are also semantic secure and privacy preserving. Furthermore, due to the randomization of $r$, the Paillier encryption can resist dictionary attacks, since the encryption of the same data will result into different ciphertexts with very high probability. This ensures that even if the adversary $\mathcal{A}$ eavesdrops the communication or even intrudes into the $\mathrm{FN}_{j}$ database and gets $C_{i j}$, he will not have access to the user's private data $D_{i j}$.

In the data aggregation phase, $\mathrm{FN}_{j}$ performs aggregation operation directly on ciphertexts. For the CC, after decryption, it only has the data sum for every dimension. Even if $\mathcal{A}$ intrudes into the $\mathrm{CC}$ database, he cannot retrieve the individual multidimensional data, i.e., $\left(m_{i 1}, m_{i 2}, \ldots, m_{i l}\right)$. Therefore, the privacy of individual data is ensured in ESMA.

Furthermore, ESMA ensures that a colluding set of users cannot compromise the privacy of other users. In fact, if $\mathcal{A}$ compromises the users' privacy, he can access the private data and retrieves the secret share $x_{i j}$. In ESMA, there is no correlation between the randomly generated shares by TA, namely, the secret shares $x_{i j} \in \mathbb{Z}_{n}^{*}$. So, compromising the secret shares of a few users will not reveal the secret shares of remaining users. Suppose the case where $\mathcal{A}$ succeeds in compromising $k_{1}-1$ users that belong to a certain $\mathrm{FN}_{j}$, and gets their secret shares $x_{1 j}, x_{2 j}, \ldots, x_{k-1 j}$. For $k_{1}-1$ users, (1) can be rewritten as follows:

$$
x_{0 j}=\sum_{i=1}^{k_{1}-1} x_{i j}+x_{k_{1 j} j} \bmod n .
$$

Data privacy of the remaining user is still maintained because $\mathcal{A}$ does not have the sum of the secret shares $x_{0 j}$ of $\mathrm{CC}$ nor the Paillier private key, thereby, $\mathcal{A}$ will not be able to compromise $x_{k_{1} j}$. Consequently, in ESMA, regardless of the number of compromised users, $\mathcal{A}$ cannot access the private data of the other users.

\section{B. Data Integrity and Authentication}

In ESMA, the BLS short signature [22] is adopted to sign the user's private data and the aggregated data. For the message $\left\{C_{i j}, \mathrm{ID}_{i j}, \mathrm{TS}, \sigma_{i j}\right\}$ sent by $\mathrm{SM}_{i j}$, the corresponding $\mathrm{FN}_{j}$ first checks $\mathrm{ID}_{i j}$ and TS and then verifies the message's integrity by checking if (7) holds. We can see that each element of the 


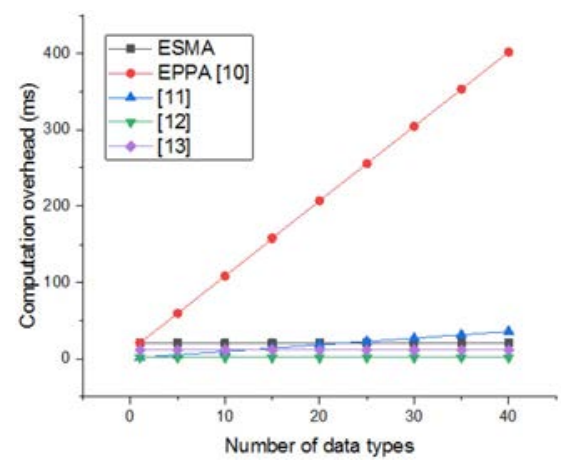

Fig. 4. Computation cost at SM.

message is involved in verification, and any manipulation of the message will cause inequality. Therefore, the message's integrity sent by $\mathrm{SM}_{i j}$ can be verified by $\mathrm{FN}_{j}$. Similarly, when receiving the message $\left\{C_{j}, \mathrm{ID}_{j}, \mathrm{TS}, \sigma_{j}\right\}$ sent by $\mathrm{FN}_{j}, \mathrm{CC}$ first checks $\mathrm{ID}_{j}$ and TS and then verifies the message's integrity by checking if (14) holds. From the equation, each element of the message is involved in verification, and any manipulation of the message will cause inequality. Therefore, the message's integrity sent by $\mathrm{FN}_{j}$ can be verified by $\mathrm{CC}$.

The signature $\sigma_{i j}$ of $\mathrm{SM}_{i j}$ is generated with the corresponding private key $s k_{i j}$, and the signature $\sigma_{j}$ of $\mathrm{FN}_{j}$ is generated with the corresponding private key $s k_{j}$. Since the adversary does not know the private keys $s k_{i j}$ and $s k_{j}$, it cannot produce the correct messages. Therefore, ESMA can guarantee that all users and their private data are legitimate.

Data integrity and authentication are then provided since the BLS signature is provably secure [22]. Consequently, $\mathcal{A}$ cannot launch active attacks without being detected. The TS included in the packet can prevent replay attacks. Any data modification or false data injection can be detected when the recipient fails to check the signature validity.

\section{ViII. Performance Evaluation}

Tonyali et al. [23] analyzed the cost generated when using $\mathrm{HE}$ in SG in terms of bandwidth and end-to-end delay. The authors state that computation and communication costs and scalability impact severely the network reliability and must be considered. Consequently, in this section, we first compare, in terms of computation and communication cost, ESMA with previous works, and then we analyze its scalability.

\section{A. Computation Cost}

Using well-known cryptographic libraries, MIRACL [24], and PBC [25], we compute the cost of the cryptographic operations used in ESMA and previous schemes. We consider for Paillier encryption a 1024-b $n$ and for pairing a base field size of 160 b. For ECC, we use the standard curve secp160r1. Table III shows the results obtained on a computer with Intel Core i5-2430 2.4-GHz CPU and 2-GB RAM. Note that multiplication in $\mathbb{Z}_{n^{2}}$, modular addition, and hash operations are negligible compared to exponentiation in $\mathbb{Z}_{n^{2}}$ and pairing operations. We compare ESMA with the schemes that achieve MDA, namely, EPPA [10], Boudia et al. [11], Ming et al. [12], and Zuo et al. [13] (see Table I).
TABLE III

COST OF CRYPTOGRAPHIC OPERATIONS

\begin{tabular}{lll}
\hline \hline Symbol & Operation & Time $(\mathrm{ms})$ \\
\hline $\mathrm{C}_{\mathrm{e}}$ & Exponentiation in $\mathbb{Z}_{n^{2}}$ & 9,78 \\
$\mathrm{C}_{\mathrm{m}}$ & Multiplication in $\mathbb{G}_{1}$ & 1,18 \\
$\mathrm{C}_{\mathrm{p}}$ & Pairing & 22,84 \\
$\mathrm{C}_{\mathrm{p}}$ & ECC Point Multiplication & 0,44 \\
$\mathrm{C}_{\mathrm{E}-\mathrm{El} \text { Gamal }}$ & El Gamal Encryption & 5,81 \\
$\mathrm{C}_{\text {D-EIGamal }}$ & El Gamal Decryption & 3,14 \\
$\mathrm{C}_{\text {exp }}$ & Exponentiation in $\mathbb{Z}_{n}$ & 2,88 \\
\hline \hline
\end{tabular}

In ESMA, when $\mathrm{SM}_{i j}$ produces its report, it requires $2 C_{e}$ to generate $C_{i j}$ and $C_{m}$ to generate $\sigma_{i j}$. In EPPA [10], $\mathrm{SM}_{i j}$ performs $(l+1) C_{e}$ and $C_{m}$ to generate the ciphertext and the signature. In [11], $\mathrm{SM}_{i j}$ performs $2(l+1) C_{p m}$. In [12], $\mathrm{SM}_{i j}$ performs $4 C_{p m}$. In [13], $\mathrm{SM}_{i j}$ performs $C_{E-E l \text { Gamal }}$ and $2 C_{\text {exp }}$. We present a comparison in Fig. 4. The results show that ESMA significantly reduces the computation cost at the user side in comparison with EPPA scheme which also utilizes Paillier to achieve MDA. In [11], the cost increases linearly with the number of DTs. In [12] and [13], the cost is efficient; however, as we will see later, this advantage is lost at CC where the decryption operation requires solving DLP which is a heavy computation in the case of large plaintexts.

In ESMA, EPPA, and [13], when $\mathrm{FN}_{j}$ (gateway in the case of EPPA and [13]) receives all of the reports from the covered $\mathrm{SM}_{i j}$, it first performs a batch verification to verify the data that needs $\left(k_{1}+1\right) C_{p}$. Then, it aggregates the ciphertexts and produces a signature, which consists of several negligible multiplications in $\mathbb{Z}_{n^{2}}$ and $C_{m}$ (multiplications in $\mathbb{Z}_{n}$ and $C_{\text {exp }}$ in [13]). Consequently, at $\mathrm{FN}_{j}$, the costs are almost the same, except for [11] and [12], which employ an Elliptic curvebased batch verification that is more efficient than pairing one. However, in the fog computing paradigm, FNs are considered to have more computational abilities than traditional gateways. Thus, the aggregation operation can be efficiently computed.

In ESMA and EPPA [10], when CC (OA in the case of EPPA) receives a packet from $\mathrm{FN}_{j}$, it first performs $2 C_{p}$ to verify the data and then retrieves the aggregation result by performing $C_{e}$. In [11], the CC performs $3 C_{p m}$ and $l$ times a reverse mapping function which requires solving the ECDLP to recover $M$. To verify and retrieve the data in [12], the CC performs $4 C_{p m}$ and a computation of the ECDLP to recover $M$, while in [13], the CC performs $2\left(k_{1}+1\right) C_{p}, C_{\mathrm{D}-\mathrm{ElGamal}}$ and a computation of the DLP to recover $M$. By considering Pollard's lambda method to compute DLP and ECDLP, we conduct experiments to compute the execution time at CC using MIRACL. The method requires a time complexity of $O\left(l \cdot \sqrt{k_{1} \cdot 2^{z}}\right), O\left(\sqrt{l \cdot k_{1} \cdot 2^{z}}\right), O\left(\sqrt{(l+1) \cdot k_{1} \cdot 2^{z}}\right)$ in [11]-[13], respectively. The results presented in Fig. 5 show that regardless of the size of the aggregation result $M$, EPPA and ESMA have the same computation overhead at CC. However, in the other works, the higher is the size of the aggregation result, the higher is the computation time. In fact, an aggregate size higher than $40 \mathrm{~b}$ cannot be efficiently recovered at CC at all. Ugus et al. [26] stated that in the El Gamal additive encryption scheme, the final aggregation must be small enough for realistic applications (e.g., 3 bytes) allowing 


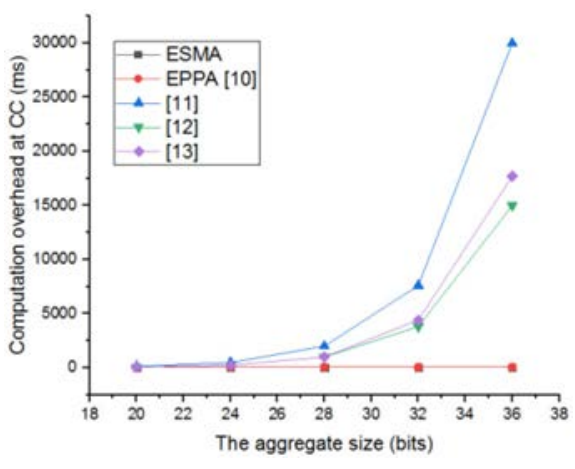

Fig. 5. Computation cost at CC vs. The aggregate size.

a successful recovery at the decryption device. Consequently, the schemes of Boudia et al. [11], Ming et al. [12], and Zuo et al. [13] cannot be practical for large aggregation results for SG. The super increasing sequence has been utilized in [12] and [13] with El Gamal HE to achieve MDA. In these two schemes, the CC has to solve the DLP to retrieve the aggregated data. Boudia et al. [11] used El Gamal HE with multiple public keys. However, in this case, also, the CC has to compute the ECDLP to retrieve the aggregated data. In the aforementioned schemes, the authors use heavy computation either to encrypt [10], [11] or to decrypt [11]-[13] the multidimensional data. Consequently, ESMA is the most efficient.

In 2019, Saleem et al. [18] have proposed a fault-tolerant mechanism to achieve resilience to node failures in their ODA scheme for FCSG. However, in their proposal, the faulttolerant decryption cost increases at $\mathrm{CC}$ with the increasing number of faulty SMs. In fact, the more are the number of faulty SMs more cryptographic operations are executed at CC. Furthermore, the decryption at $\mathrm{CC}$ requires solving DLP to recover the aggregation result. In ESMA, regardless of the number of faulty SMs, the cost of the fault-tolerant decryption at $\mathrm{CC}$ is always the same. Fig. 6 shows the efficiency of our fault-tolerant decryption compared to that of Saleem et al. [18].

\section{B. Communication Cost}

In MDA schemes, $l$ types of data are encrypted and sent by each SM to the corresponding aggregator. The communication overhead can be divided into two parts: 1) SM-to-FN communication and 2) FN-to-CC communication. In ESMA, $C_{i j}$, $\mathrm{ID}_{i j}$, TS, and $\sigma_{i j}$ are sent from SM to $\mathrm{FN}_{j}$, where $C_{i j} \in \mathbb{Z}_{n^{2}}$ and $\sigma_{i j} \in \mathbb{G}_{1}$. Let the size of $\mathrm{ID}_{i j}$ and TS be all $8 \mathrm{~B}$. Therefore, the communication cost SM-to-FN is $2048+64$ $+160=2272$ b. Next, we analyze FN-to-CC communication. In ESMA, $\left\{C_{j}, \mathrm{ID}_{j}, \mathrm{TS}\right.$, and $\left.\sigma_{j}\right\}$ are sent from $\mathrm{FN}_{j}$ to $\mathrm{CC}$, where $C_{i j} \in \mathbb{Z}_{n}^{2}$ and $\sigma_{i j} \in \mathbb{G}_{1}$. Therefore, the communication cost FN-to-CC is $2048+64+160=2272$ b. The comparison of communication cost with previous MDA schemes is shown in Table IV.

We depict in Fig. 7 the comparison in terms of communication cost SM-to-FN for 20 types of data. It can be seen that ESMA incurs lower communication overhead than [11].
TABLE IV

COMMUNICATION OVERHEAD COMPARISON

\begin{tabular}{ccc}
\hline \hline Scheme & SM-to-FN & FN-to-CC \\
\hline Lu et al. [10] & 2308 bits & 2308 bits \\
Merad et al. [11] & $160 l+544$ bits & $160 l+544$ bits \\
Ming et al. [12] & 704 bits & 704 bits \\
Zuo et al. [13] & 2112 bits & 2112 bits \\
ESMA & 2272 bits & 2272 bits \\
\hline \hline
\end{tabular}

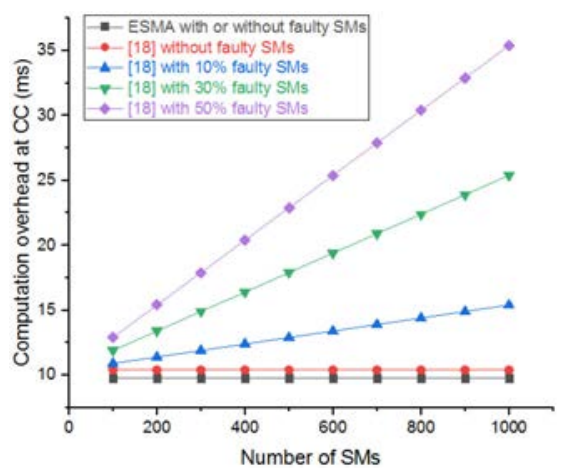

Fig. 6. FT decryption cost at CC.

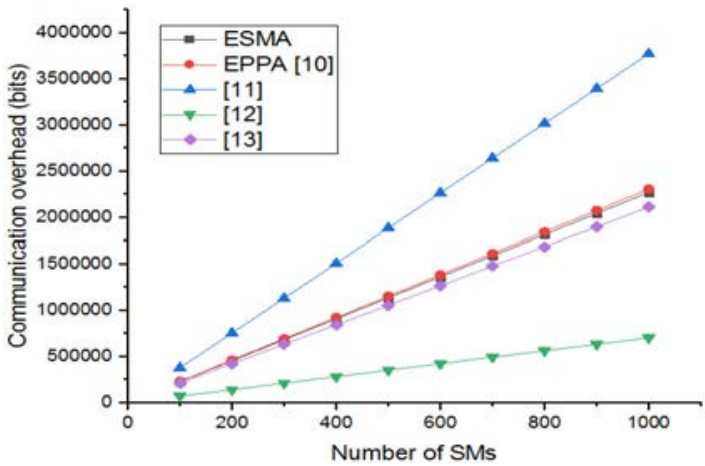

Fig. 7. SM-to-FN communication cost.

The cost of communication is approximately equal to that of [10] and [13]. However, in comparison with [9], the communication overhead is higher in ESMA. The reason is that Ming et al. [12] employed in their scheme elliptic curve groups for encryption and signature, which leads to cost-efficient communications. Nevertheless, first, as previously mentioned, the main drawback of their proposal is that the CC has to compute the ECDLP, which severely limits the upper bound. Second, they consider a finite field with a 160-b $q$, the plaintext space is then severely limited for the considered super-increasing sequence, which has an impact on the number of DTs as well. The same observation can be made for [11] and [13]. In ESMA, there is no extra computation at SM and CC. The plaintext is up to $1024 \mathrm{~b}$ and an important number of DTs can be supported in a single ciphertext.

\section{Scalability Analysis}

In ESMA, we use an encoding function to structure the multidimensional data in $\mathbb{Z}_{n}$. In our implementation, we consider a 1024-b $n$ in Paillier encryption. However, Paillier can support larger key sizes, such as 2048 and $4096 \mathrm{~b}$ for improved 
TABLE V

SCALABILITY ANALYSIS

\begin{tabular}{cccc}
\hline \hline $\mathrm{n} \mid$ & $\mathrm{z}$ & $\mathrm{k}_{1}$ & $l$ \\
\hline 1024 & 16 bits & 125 & 44 \\
& & 250 & 42 \\
& & 500 & 40 \\
& \multirow{3}{*}{32 bits } & 1000 & 39 \\
& & 125 & 26 \\
& & 500 & 25 \\
& & 1000 & 24 \\
\hline \hline
\end{tabular}

TABLE VI

STORAGE OVERHEAD COMPARISON

\begin{tabular}{ccc}
\hline \hline Scheme & SM & FN \\
\hline Lu et al. [10] & $1024(l+1)+192$ bits & $320 k_{l}+192$ bits \\
Merad et al. [11] & $320(l+1)+192$ bits & $320\left(k_{1}+1\right)+192$ bits \\
Ming et al. [12] & $992+l\left|\mathrm{a}_{\mathrm{i}}\right|$ bits & $320\left(k_{l}+1\right)+192$ bits \\
Zuo et al. [13] & $1568+l\left|\mathrm{a}_{\mathrm{i}}\right|$ bits & $512 k_{1}+192 \mathrm{bits}$ \\
ESMA & 3264 bits & $320 k_{l}+192$ bits \\
\hline \hline
\end{tabular}

security. Recall that each $\mathrm{SM}_{i j}$ encodes its $l$ types of data $\left(m_{i 1}, m_{i 2}, \ldots, m_{i l}\right)$ into $\left(d_{i 1}, d_{i 2}, \ldots, d_{i l}\right)$ as

$$
\begin{aligned}
d_{i k} & =\left(m_{i k}\right)_{2} \| 0^{\theta}, k=1, \ldots, l \\
\text { where } \theta & =\left(\left\lceil\log _{2}\left(k_{1}\right)\right\rceil+z\right) *(k-1) .
\end{aligned}
$$

In Table $\mathrm{V}$, we show, according to $n, z$, and $k_{1}$, the number of DTs $l$ that can be supported in a single ciphertext. The table shows that using our encoding function, an important number of DTs $l$ can be supported in a single Paillier ciphertext. For instance, with $z=16 \mathrm{~b}$ and 500 reporting SMs, 40 DTs can be supported in a single ciphertext. Note that the number $z$ can be significantly reduced with a reference technique, such as presented in [27] for efficient communications in sensor networks [28]. The plaintext space in previous works cannot support a large number of DT as mentioned earlier.

\section{Storage Analysis}

In ESMA, each SM stores in its memory its ID, the public key of CC, a private key, and a secret share. Each FN stores its ID, the public key of each covered SM, and a private key. In EPPA [10], each SM stores its ID, $l$ generators, $n$, and a private key. Each FN stores the public key of each covered SM and a private key. In [11], each SM stores its ID, $l$ public keys, a generator $G$, and a private key. Each FN stores the public key of each covered SM and a private key. In [12], each SM stores its ID, $l$ big primes $a_{i}$, the CC's public keys, a generator $P$, a secret share, and a private key. Each FN stores the public key of each covered SM, $P$, and a private key. In [13], each SM stores its ID, the CC's public key, $l$ big primes $\mathrm{a}_{i}, g$, and a private key. Each FN stores the public key of each covered SM and a private key. A comparison with previous works is presented in Table VI. Note that the points on the curve are considered in their uncompressed form. The main observation we can make is that the storage overhead in previous schemes depends on the number of DTs $l$. In our work, the overhead is $3264 \mathrm{~b}$ whatever the number $l$.

\section{CONCLUSION}

In this article, we proposed ESMA, an efficient and secure MDA for FCSG. ESMA can structure and encrypt the multidimensional data into a single Paillier ciphertext. Thanks to the encoding function employed, which also gives the ability to the $\mathrm{CC}$ to recover the aggregated data for every dimension efficiently and securely. The security analysis shows that the privacy, confidentiality, integrity, and authentication of the data are provided. The performance analysis shows the scalability advantage of ESMA, the efficiency of our fault-tolerant mechanism, and also, the cost-efficiency of ESMA in terms of computation and communication. ESMA can also be adapted to respond to other queries than summation. Therefore, it satisfies the application needs of SG for smart cities.

\section{REFERENCES}

[1] G. Dileep, "A survey on smart grid technologies and applications," Renew. Energy, vol. 146, pp. 2589-2625, Feb. 2020.

[2] S. Kaneriya et al., "Data consumption-aware load forecasting scheme for smart grid systems," in Proc. IEEE Globecom Workshops, 2018, pp. $1-6$.

[3] S. Tanwar, S. Kaneriya, N. Kumar, and S. Zeadally, "ElectroBlocks: A blockchain-based energy trading scheme for smart grid systems," Int. J. Commun. Syst., vol. 33, no. 15, 2020, Art. no. e4547.

[4] A. Kumari, D. Vekaria, R. Gupta, and S. Tanwar, "Redills: Deep learning-based secure data analytic framework for smart grid systems," in Proc. IEEE ICC Workshops, 2020, pp. 1-6.

[5] M. Shateri, F. Messina, P. Piantanida, and F. Labeau, "Real-time privacypreserving data release for smart meters," IEEE Trans. Smart Grid, vol. 11, no. 6, pp. 5174-5183, Nov. 2020.

[6] F. Li, B. Luo, and P. Liu, "Secure information aggregation for smart grids using homomorphic encryption," in Proc. IEEE SmartGridComm, 2010, pp. 327-332.

[7] A. Kumari, S. Tanwar, S. Tyagi, N. Kumar, M. S. Obaidat, and J. J. P. C. Rodrigues, "Fog computing for smart grid systems in the $5 \mathrm{G}$ environment: Challenges and solutions," IEEE Wireless Commun., vol. 26, no. 3, pp. 47-53, Jun. 2019.

[8] M. S. H. Nazmudeen, A. T. Wan, and S. M. Buhari, "Improved throughput for power line communication (PLC) for smart meters using fog computing based data aggregation approach," in Proc. IEEE Int. Smart Cities Conf., 2016, pp. 1-4.

[9] L. Lyu, K. Nandakumar, B. Rubinstein, J. Jin, J. Bedo, and M. Palaniswami, "PPFA: Privacy preserving fog-enabled aggregation in smart grid," IEEE Trans. Ind. Informat., vol. 14, no. 8, pp. 3733-3744, Aug. 2018.

[10] R. Lu, X. Liang, X. Li, X. Lin, and X. Shen, "EPPA: An efficient and privacy-preserving aggregation scheme for secure smart grid communications," IEEE Trans. Parallel Distrib. Syst., vol. 23, no. 9, pp. 1621-1631, Sep. 2012.

[11] O. R. M. Boudia, S. M. Senouci, and M. Feham, "Elliptic curve-based secure multidimensional aggregation for smart grid communications," IEEE Sensors J., vol. 17, no. 23, pp. 7750-7757, Dec. 2017.

[12] Y. Ming, X. Zhang, and X. Shen, "Efficient privacy-preserving multidimensional data aggregation scheme in smart grid," IEEE Access, vol. 7, pp. 32907-32921, 2019.

[13] X. Zuo, L. Li, H. Peng, S. Luo, and Y. Yang, "Privacy-preserving multidimensional data aggregation scheme without trusted authority in smart grid," IEEE Syst. J., early access, Jun. 16, 2020, doi: 10.1109/JSYST.2020.2994363.

[14] M. Badra and S. Zeadally, "Lightweight and efficient privacy-preserving data aggregation approach for the smart grid," Ad Hoc Netw., vol. 64, pp. 32-40, Sep. 2017.

[15] H. Shen, Y. Liu, Z. Xia, and M. Zhang, "An efficient aggregation scheme resisting on malicious data mining attacks for smart grid," Inf. Sci., vol. 526, pp. 289-300, Jul. 2020.

[16] J.-N. Liu, J. Weng, A. Yang, Y. Chen, and X. Lin, "Enabling efficient and privacy-preserving aggregation communication and function query for fog computing based smart grid," IEEE Trans. Smart Grid, vol. 11, no. 1 , pp. 247-257, Jan. 2020. 
[17] F. Y. Okay, S. Ozdemir, and Y. Xiao, "Fog computing-based privacy preserving data aggregation protocols," Trans. Emerg. Telecommun. Technol., vol. 31, no. 4, 2020, Art. no. e3900.

[18] A. Saleem et al., "FESDA: Fog-enabled secure data aggregation in smart grid IoT network," IEEE Internet Things J., vol. 7, no. 7, pp. 6132-6142, Jul. 2020.

[19] S. Zhao et al., "Smart and practical privacy-preserving data aggregation for fog-based smart grids," IEEE Trans. Inf. Forensics Security, vol. 16, pp. 521-536, Aug. 2020.

[20] P. Paillier, "Public-key cryptosystems based on composite degree residuosity classes," in Proc. Int. Conf. Theory Appl. Cryptograph. Technol., 1999, pp. 223-238.

[21] S. Li, K. Xue, Q. Yang, and P. Hong, "PPMA: Privacy-preserving multisubset data aggregation in smart grid," IEEE Trans. Ind. Informat., vol. 14, no. 2, pp. 462-471, Feb. 2018.

[22] D. Boneh, C. Gentry, B. Lynn, and H. Shacham, "Aggregate and verifiably encrypted signatures from bilinear maps," in Proc. Int. Conf. Theory Appl. Cryptograph. Technol., 2003, pp. 416-432.

[23] S. Tonyali, R. Munoz, K. Akkaya, and U. Ozgur, "A realistic performance evaluation of privacy-preserving protocols for smart grid AMI networks," J. Netw. Comput. Appl., vol. 119, pp. 24-41, Oct. 2018.

[24] Certivox. (2014). Multiprecision Integer and Rational Arithmetic C/C++ Library (MIRACL). [Online]. Available: https://github.com/ miracl/MIRACL

[25] B. Lynn. PBC Library. Accessed: Jun. 14, 2013. [Online]. Available: https://crypto.stanford.edu/pbc/

[26] O. Ugus et al., "Optimized implementation of elliptic curve-based additive homomorphic encryption for wireless sensor networks," 2009. [Online]. Available: arXiv:0903.3900.

[27] H. Sanli, S. Ozdemir, and H. Cam, "SRDA: Secure reference-based data aggregation protocol for wireless sensor networks," in Proc. IEEE 60th Int. Conf. Veh. Technol. (VTC-Fall), vol. 7, Sep. 2004 pp. 4650-4654.

[28] H. Fouchal, Y. Francillette, P. Hunel, and N. Vidot, "A distributed power management optimisation in wireless sensors networks," in Proc. $L C N$, 2009 , pp. $763-769$. 